\title{
nuevas interpretaciones de antiguos conceptos sobre el terreno como soporte de firmes blancos
}

Es sabido cómo el terreno, a efectos de su aplicación en carreteras, se caracteriza por una serie de cualidades, representables por parámetros cuya determinación es siempre experimental (y, por lo tanto, sujeta a la influencia del método de ensayo), y cuya correlación con el comportamiento posterior en obra no es siempre muy clara.

¿Significa, por ejemplo, que un terreno presenta una estabilidad máxima cuando su densidad en seco es máxima? No siempre se cumple esta correlación. Y, además, ¿qué es estabilidad, qué características cualitativas y cuantitativas la definen? ¿Qué relación tiene el ensayo de penetración CBR con la capacidad resistente del suelo? ¿Qué se entiende por capacidad resistente?

La técnica moderna abunda en prescripciones de tipo empírico, sancionadas muchas veces por una experiencia a escala natural. Pero al variar los datos ambientales (tales como la clase de terreno o las caracteristicas del clima), pueüe no ser lícito extrapolar las conclusiones de aquella experiencia. Surge entonces la idea de que, como en otras ramas de la Técnica más antiguas y trilladas (no debe olvidarse que la Mecánica del Suelo, como tal, apenas tiene treinta y cinco años de vida), los criterios empíricos primitivos resultan luego ser casos particulares de criterios más generales basados en una teoría orgánica, que tiene en cuenta todas las circunstancias del problema. Pero ocurre que, cuando se aborda el problema por el camino teórico, el número de variables a considerar es tal, que se tropieza en seguida con dificultades de tipo matemático, que obligan a establecer hipótesis simpliflcatorias que, acumulándose, alejan cada vez más el resultado de los ensayos del resultado de la teoría... Muchas veces no es válido el principio de superposición, que permite estudiar cómodamente cada causa y su correspondiente efecto, independientemente de las demás.

La penosa labor del investigador consiste, ante este problema tan complejo, en discriminar cuáles son, cualitativamente, las variables que intervienen en el fenómeno, y determinar, por medio de ensayos simplificados, pero representativos, su valor cuantitativo y su correlación con el comportamiento real del terreno en obra.

Este método se puede llamar semi-empírico, por cuanto la determinación de variables debe ajustarse a un esquema lógico: a una teoría; pero su determinación cuantitativa se basa en ensayos que, a veces, poco tienen que ver con las circunstancias reales de la obra.

Teniendo muy presentes las consideraciones anteriores, se ha intentado en las páginas siguientes describir algunos conceptos ya familiares al ingeniero, bajo una perspectiva diferente: con vistas a una más general visión de conjunto, que, indudablemente, la investigación hará sazonar en un plazo no muy largo. Estas nuevas interpretaciones son una a modo de herramienta para el trabajo técnico: no son, ni pretenden ser, la solución definitiva de ninguno de los muchos problemas que la técnica de la carretera tiene planteados. 


\section{El diagrama de estado de un suelo}

Dejando a un lado consideraciones de origen petrográfico o mineralógico, resulta que un suelo es una mezcla de partículas de diversos tamaños, a la cual son aplicables las teorías de la Estadística sobre conjuntos ordenados. Lo primero que cabe es distribuir estas partículas por tamaños, mediante un análisis granulométrico.

La experiencia ha demostrado que en muchas de las propiedades de un suelo tiene sentido desglosar éste en dos fracciones:

Los áridos gruesos, por encima de $2 \mathrm{~mm}$ de diámetro.

El suelo propiamente dicho, por debajo de $2 \mathrm{~mm}$ de diámetro.

Los áridos gruesos tienen más que nada una función pasiva, inerte; y se puede estudiar cómodamente la influencia que en las propiedades del suelo tiene una determinada proporción de áridos gruesos, con independencia casi total de su composición; por lo que, en lo siguiente, sólo consideraré el suelo propiamente dicho, con tamaño máximo de 2 milímetros.

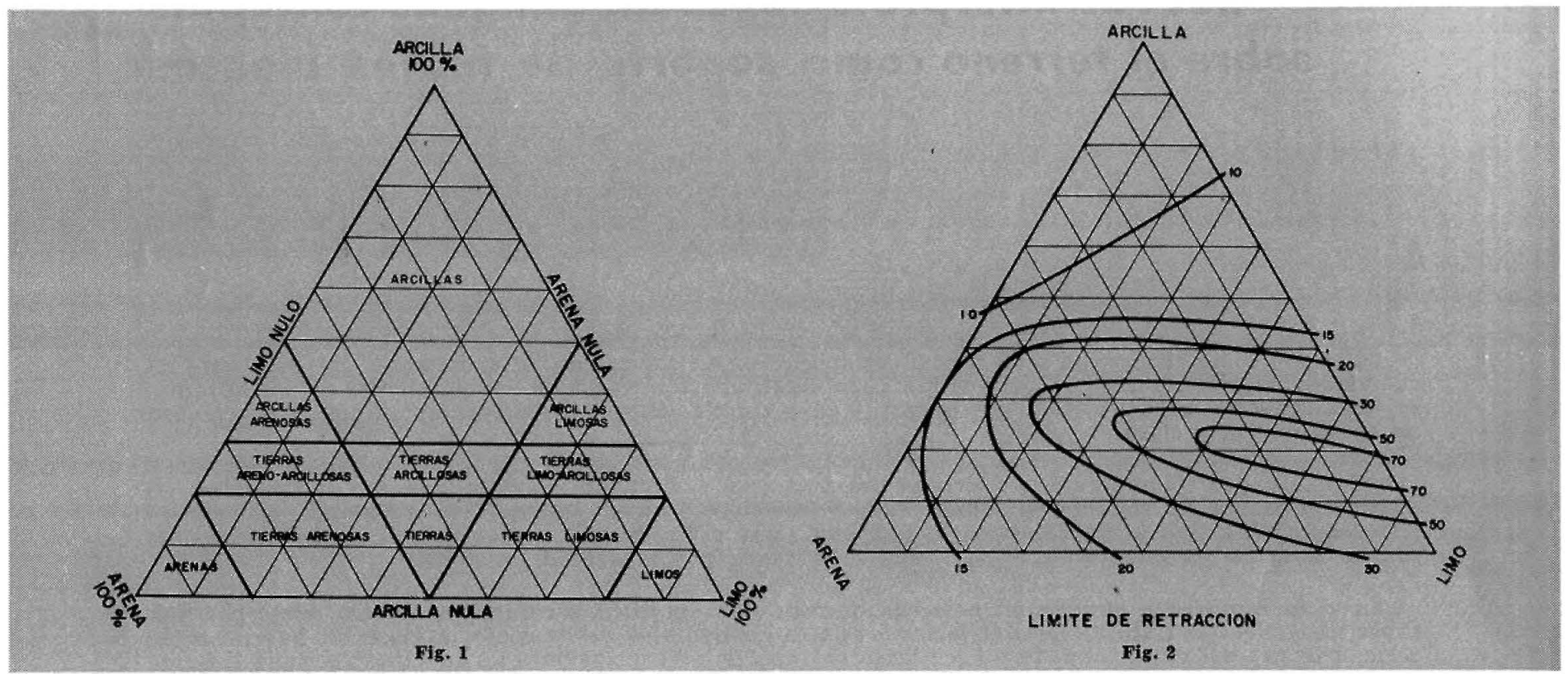

A su vez, en el suelo así definido pueden considerarse tres fracciones granulométricas, netamente diferenciadas:

La arena, sin cohesión, pero con rozamiento interno; muy permeable, pero con escasa capilaridad: de 2 a 0,06 milímetros.

El limo, intermedio en tamaño entre la arena y la arcilla; poco cohesivo, muy capilar y bastante permeable.

La arcilla, cohesiva, plástica y casi impermeable; menos capilar que el limo, pero más que la arena; menor de 0,002 milímetros.

La clasificación anterior se basa en la del MIT, y es válida en rasgos generales para todo tipo de suelo.

Resulta, por lo tanto, indicada la utilización de un gráfico en coordenadas triangulares (fig. 1) en el que se indiquen los porcentajes relativos de arena, limo y arcilla, junto con la propiedad del suelo, cuya variación con las proporciones relativas de los componentes del suelo conviene averiguar.

Esto es particularmente ventajoso cuando se trata de mejorar ciertas características de un suelo por medio de un material de aportación, que puede suplir, por ejemplo, una fracción granulométrica ausente. Si se trata de dos suelos, éstos estarán representados por dos puntos en el diagrama triangular, a los que corresponderán unos valores de la tercera variable (representada por medio de "curvas de 
nivel"). La mezcla entre los dos suelos se situará sobre la recta que une dichos dos puntos representativos, a distancias de ellos proporcionales a la cantidad relativa de uno y otro en la mezcla; la tercera variable queda automáticamente determinada por la "curva de nivel" correspondiente al punto representativo de la mezcla (fig. 2).

En su estado natural, el terreno contiene, además, una proporción de huecos, variable según su estado de consolidación, $\mathrm{y}$ que pueden ser llenados por el aire o por el agua, o por ambos a la vez. Sabido es que en el primer caso se dice que el suelo está seco, y se define una "densidad en seco", que depende del peso específico de las partículas de suelo, y del índice de huecos que corresponde a un estado de consolidación dado. A medida que el contenido de humedad (expresado convencionalmente en porcentaje de la densidad en seco) aumenta, va aumentando también la densidad "húmeda" del suelo, hasta llegar al estado de saturación, correspondiente a que todos los huecos han sido llenados por el agua.

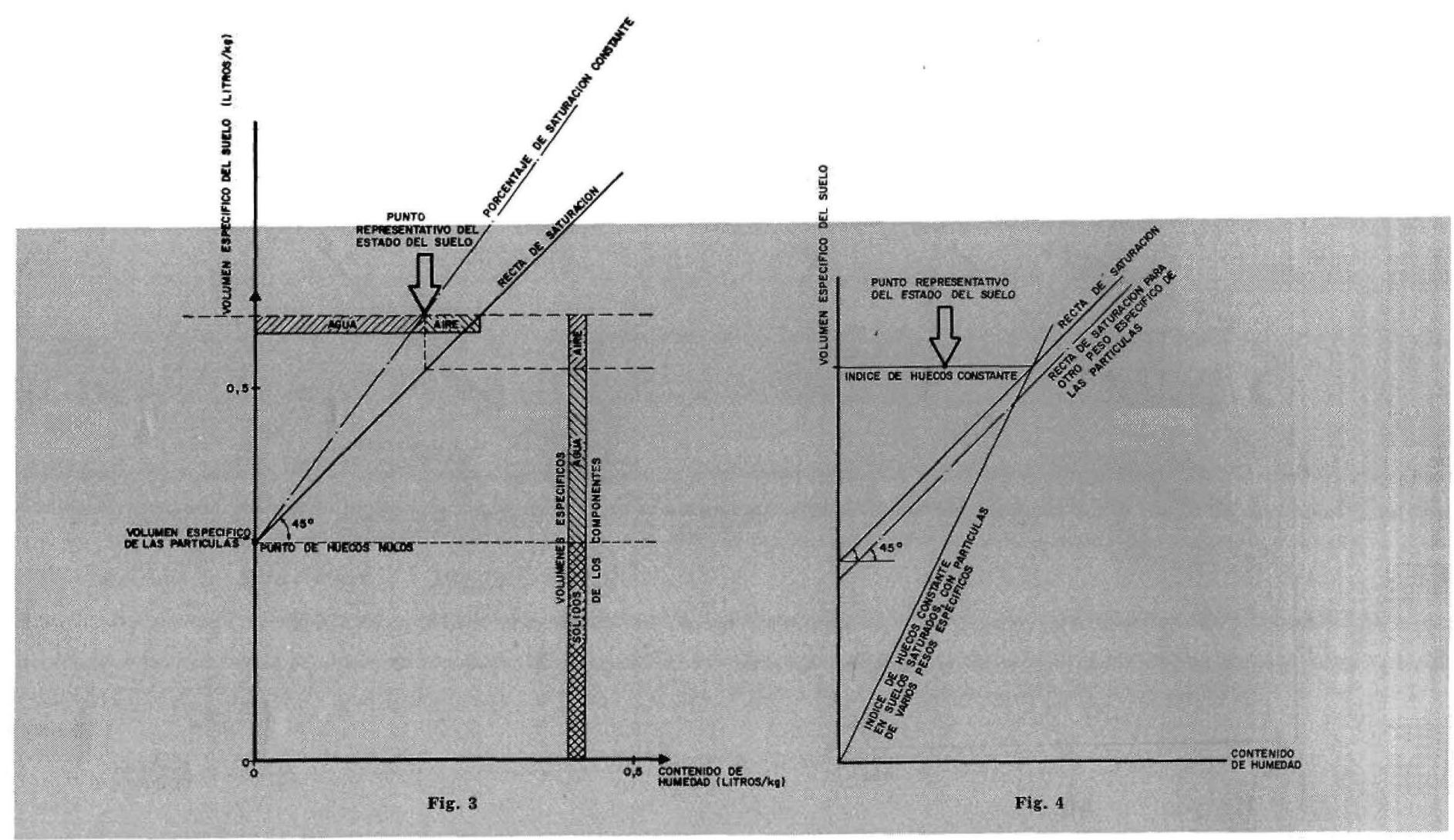

Las variables independientes que intervienen en el fenómeno son, como se ha visto, tres: la densidad "húmeda" o "en seco", el contenido de humedad, y el peso específico de las partículas. Este último es fijo para un suelo dado, cualquiera que sea su estado de consolidación y su contenido de humedad. Resulta, además, conveniente el expresar la variable "densidad", bajo su forma inversa, "volumen específico", puesto que este volumen específico referido al peso seco es independiente del grado de saturación del suelo: el volumen específico es el mismo, ya estén los poros totalmente llenos de aire (densidad en seco), parcialmente rellenos de agua (densidad húmeda) o totalmente saturados. Por otro lado, las dos variables: volumen específico y contenido de humedad (referido a un peso en seco) son homogéneas, expresándose en las mismas unidades, litros $/ \mathrm{kg}$.

Es, por lo tanto, factible el establecer un gráfico en coordenadas cartesianas volumen especifico-contenido de humedad, en el que el estado de saturación esté representado por una recta paralela a la bisectriz del primer cuadrante, y que pasa por el punto correspondiente a humedad nula, y volumen específico inverso del peso específico de las partículas de suelo (fig. 3). Este punto representaría un estado del suelo en que careciera de huecos. Si ahora se admite una cierta cantidad de huecos, y éstos están saturados de agua, el aumento de volumen específico corresponde al aumento en contenido de humedad (puesto que el volumen específico del agua es la unidad) y el punto representativo del estado del suelo se desplaza por una recta a $45^{\circ}$ con el eje, que es la "recta del suelo saturado". Si, además, hay huecos ocupados por el aire, aumenta el volumen específico del suelo sin aumentar su humedad: los estados posibles se hallan por encima de la recta de suelos saturados. Es fácil demostrar que la proporción agua-aire en volumen está dada por la relación de las distancias del punto repre- 
sentativo del estado del suelo a las rectas de humedad nula y de saturación, contadas sobre un segmento horizontal; $\mathrm{y}$ consecuentemente, los estados con igual porcentaje de saturación se hallan sobre una recta que pasa por el punto de "huecos nulos".

Resulta aparente en la figura 3 la manera de apreciar de un solo golpe la proporción (en volumen) ocupada por los sólidos, el agua y el aire. El grado de saturación se puede tener ya directamente sin más que medir la inclinación de la "recta de porcentaje de saturación constante" sobre una escala aproplada (válida para cualquier "punto de huecos nulos"). El índice de huecos es constante para los puntos situados en una misma horizontal (volumen especiffco constante), pero depende de la posición de la "recta de saturación", sobre la cual se puede ya tener acotada la escala de índice de huecos. La variación con el peso específlco del suelo, de los puntos acotados en "índice de huecos" sobre la "recta de saturación", se veriflea a lo largo de una recta que pasa por el origen de coordenadas, como resulta fácll demostrar (fig. 4).

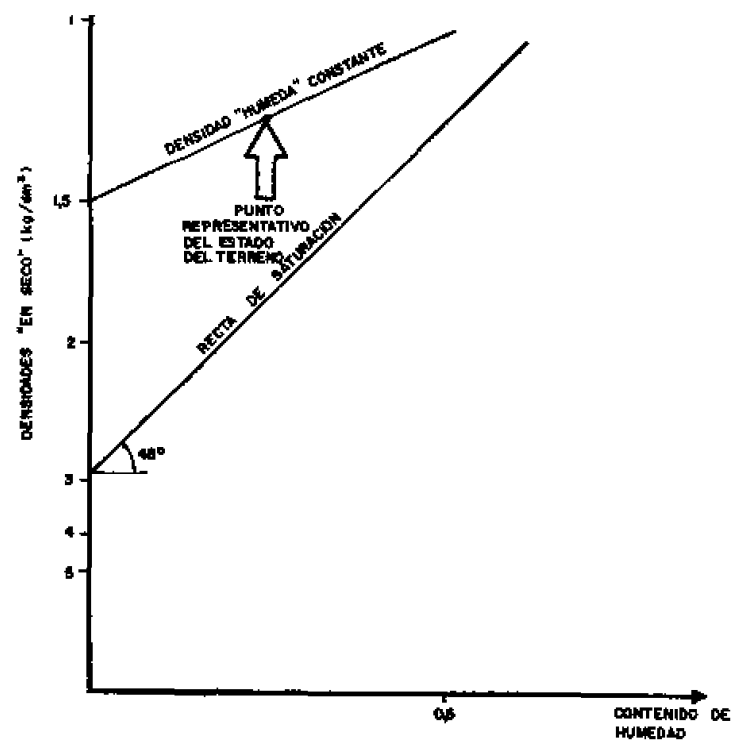

Fiz. 5

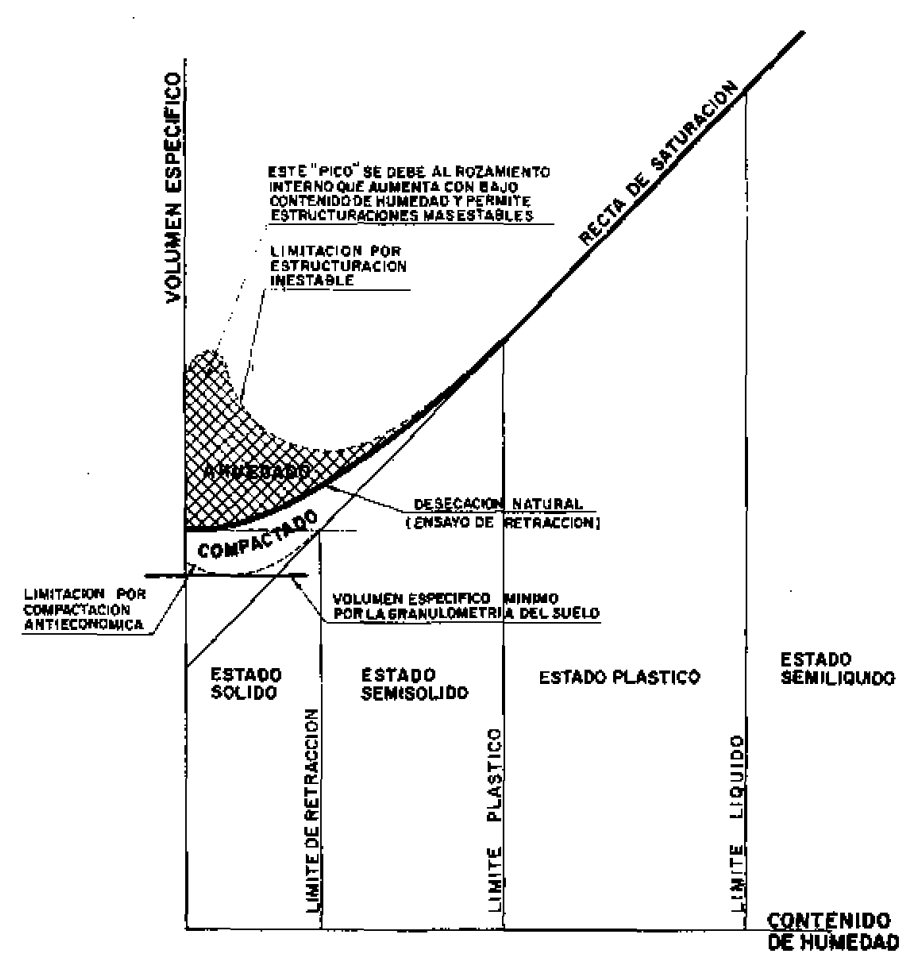

Fig. 6

Las densidades en seco se obtienen directamente hallando la inversa del volumen especiflco, y se acotan en la escala de ordenadas, pues es más frecuente manejar densidađes que volúmenes; la escala, claro está, no es lineal, sino hiperbólica. Las densidades "húmedas" constantes están representadas por rectas de inclinación variable defintdas por:

$$
d_{\mathrm{h}}=d_{\mathrm{a}}(1+h)
$$

siendo:

$$
\begin{aligned}
& h=\text { contenido de humedad, } \\
& d_{\mathrm{a}}=\text { densidad "en seco". }
\end{aligned}
$$

Asi, pues, en el diagrama de estado trazado con coordenadas cartesianas lineales volumen específico del suelo-contenido de humedad referido al peso "en seco", se pueden determinar, además, automátíleamente, el grado de saturación, el indice de huecos, la densidad "en seco" y la densidad "húmeda" o total; y lo que es más importante, se aprecia de forma intuitiva las relaciones de las variables entre sí, $y$ el efecto que en las demás tiene la variación de una cualquiera de ellas.

Así, por ejemplo, el asiento o consolidación de un suelo no saturado, sin drenaje, está representado por un segmento vertical, límitado necesariamente por la recta de saturación; la consolidación de las 
arcillas suele efectuarse a lo largo de la recta de saturación. Observese que la diferencia de ordenadas (volúmenes específlcos) es proporcional al asiento de una capa; y si esta diferencia se mide en centímetros, el asiento, expresado por ese número de centímetros, corresponde a una capa euyo espesor es tal, que su peso en toneladas por metro cuadrado resuita igual a la longitud de la unidad de volúmenes específicos, expresada en metros. Por ejemplo, si a $1 \mathrm{dm}^{3} / \mathrm{kg}$ corresponden en el gráflco $50 \mathrm{~cm}$, y el cambio de estado del suelo entraña una diferencia de ordenadas de $1 \mathrm{~cm}$, corresponderá un asiento de $1 \mathrm{~cm}$ a una capa de suelo que pese 0,5 toneladas/metro cuadrado.

Resulta muy ventajoso conslgnar los resultados de ensayos, en los que intervengan las variables antes citadas, en el diagrama de estado. A cada suelo corresponden en dicho diagrama una "zona posible" bastante restringida, cuyos límites y propiedades anejas pueden ser determinados por medio del ensayo.

Asi, por ejemplo (fig. 6), para contenidos de humedad superiores al límite líquido, y aún inferioses a él, el suelo se encuentra saturado siempre, y sus estados posibles se sitúan sobre la recta de saturación. Disminuyendo el contenido de humedad empieza a haber huecos ocupables por el aire, y el límite superior de la "zona posible" se despega de la recta de saturación.

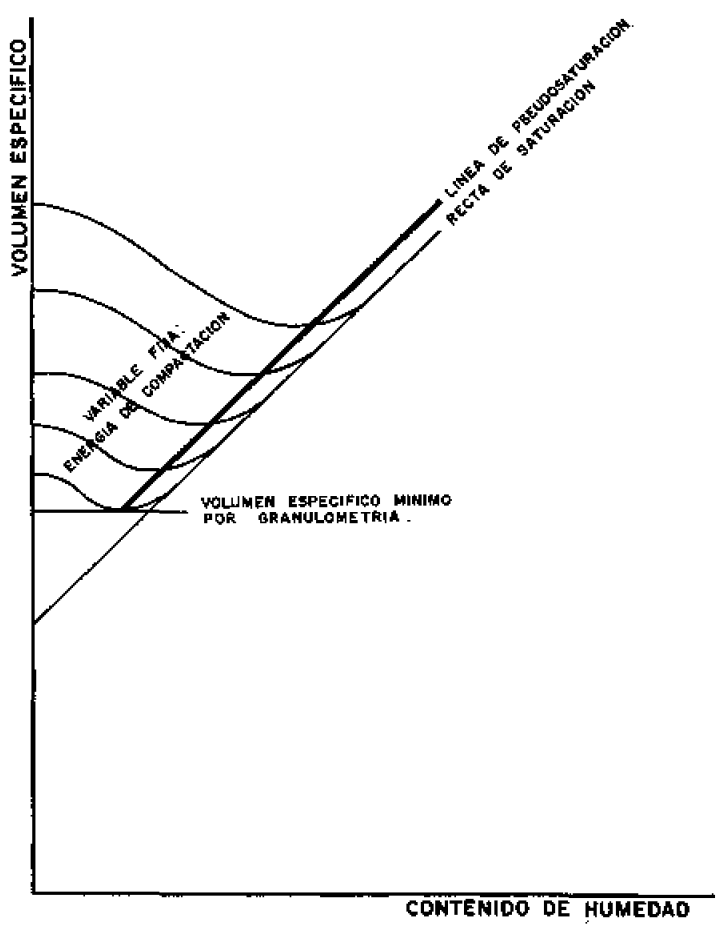

Fig. I

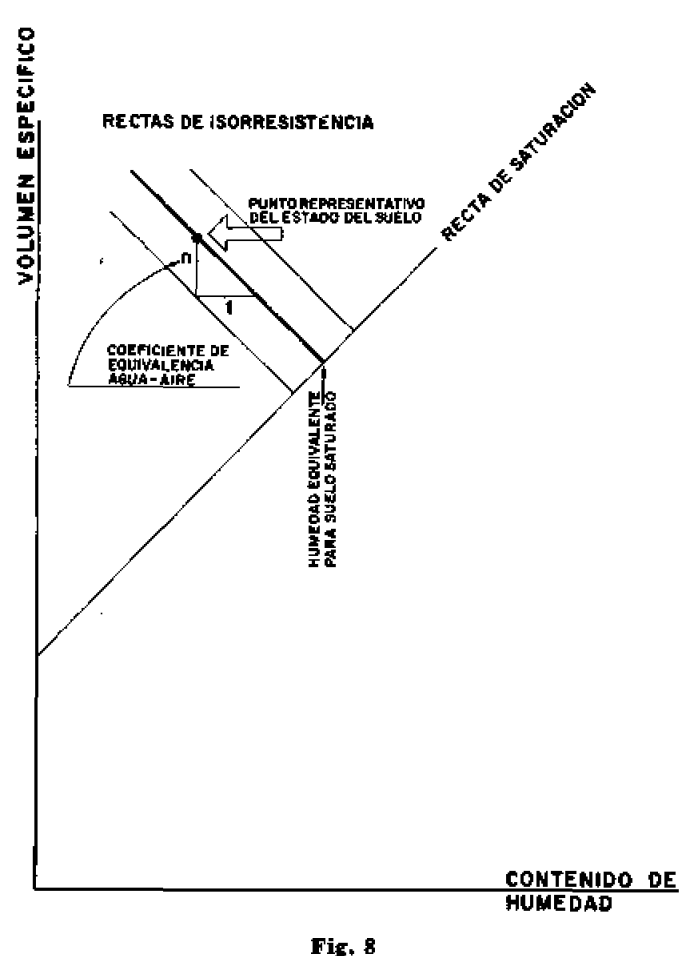

Fis. 8

Prosiguiendo la desecación hasta el límite, se alcanza una densidad máxima (volumen especifico minimo) correspondiente a una ordenación espontánea de huecos, con saturación nula: el límite de retracción se define por la intersección de la horizontal que pasa por la densidad "en seco" correspondiente, con la recta de saturación. Para bajar de ese volumen específtco mínimo se requlere una energía de compactación aportada, cada vez mayor. El límite teórico, que no se alcanza nunca, es el punto de huecos nulos.

Por otro lado, el límite superior de la zona posible está definido por huecos cada vez más ocupados por el aire, correspondientes a estructuraciones inestables de las particulas del suelo, que una compactación moderada destruge.

Los ensayos de compactación efectuados con los métodos Proctor, AASHo modificado, etc., son también susceptibles de ser representados cómodamente. La curva que Ios ingleses denominan "zero air" volds" resulta ser la recta de saturación. Ios mínimos de volumen especiflco (máxima densidad "en seco") para diferentes energías de compactación se sitúan, aproximadamente, sobre una paralela a la recta de saturación, y próxima a ella; la cual representa el volumen de aire que, por estar incluído en las películas de agua capilar, no puede ser elimtnado nor procedimientos de compactación dínámica (sin drenaje), Esta línea se llama de "pseudosaturación". 
Como, en función de la granulometría del material, se tiene un volumen específico mínimo que no se puede rebajar, la línea de pseudosaturación tiene un mínimo; aún con energía de compactación mayor, no se consiguen menores volúmenes específicos (fig. 7).

En cuanto a las propiedades resistentes, tales como CBR, módulo de reacción, resistencia a esfuerzo cortante, etc., se pueden representar por "curvas de nivel" en el diagrama de estado. Estas curvas de isorresistencia son más o menos de traza perpendicular a la recta de saturación, y con cota de resistencia creciente a medida que se acercan al punto de huecos nulos: en efecto, la resistencia de los suelos aumenta al disminuir el volumen específico y al disminuir el contenido de humedad. Algunas de estas propiedades resistentes, al menos dentro de la "zona posible", son susceptibles de representarse por una serie de rectas paralelas, que quedarían asi definidas únicamente por dos parámetros (fig. 8). Es conveniente elegir para valor de dichos parámetros los conceptos de "humedad equivalente para suelo saturado", es decir, el punto de intersección de la recta isorresistente con la recta de saturación; y el de "equivalencia agua-aire", que representa la pendiente de la recta, aumentada en una unidad (la pendiente se considera positiva). Quiere decirse con esto último que si la pendiente de la recta isorresistente es $n$, la resistencia correspcndiente a un cierto estado del suelo no se altera si se le restan $n+1$ decímetros cúbicos de aire y se le añade $1 \mathrm{dm}^{3}$ de agua.

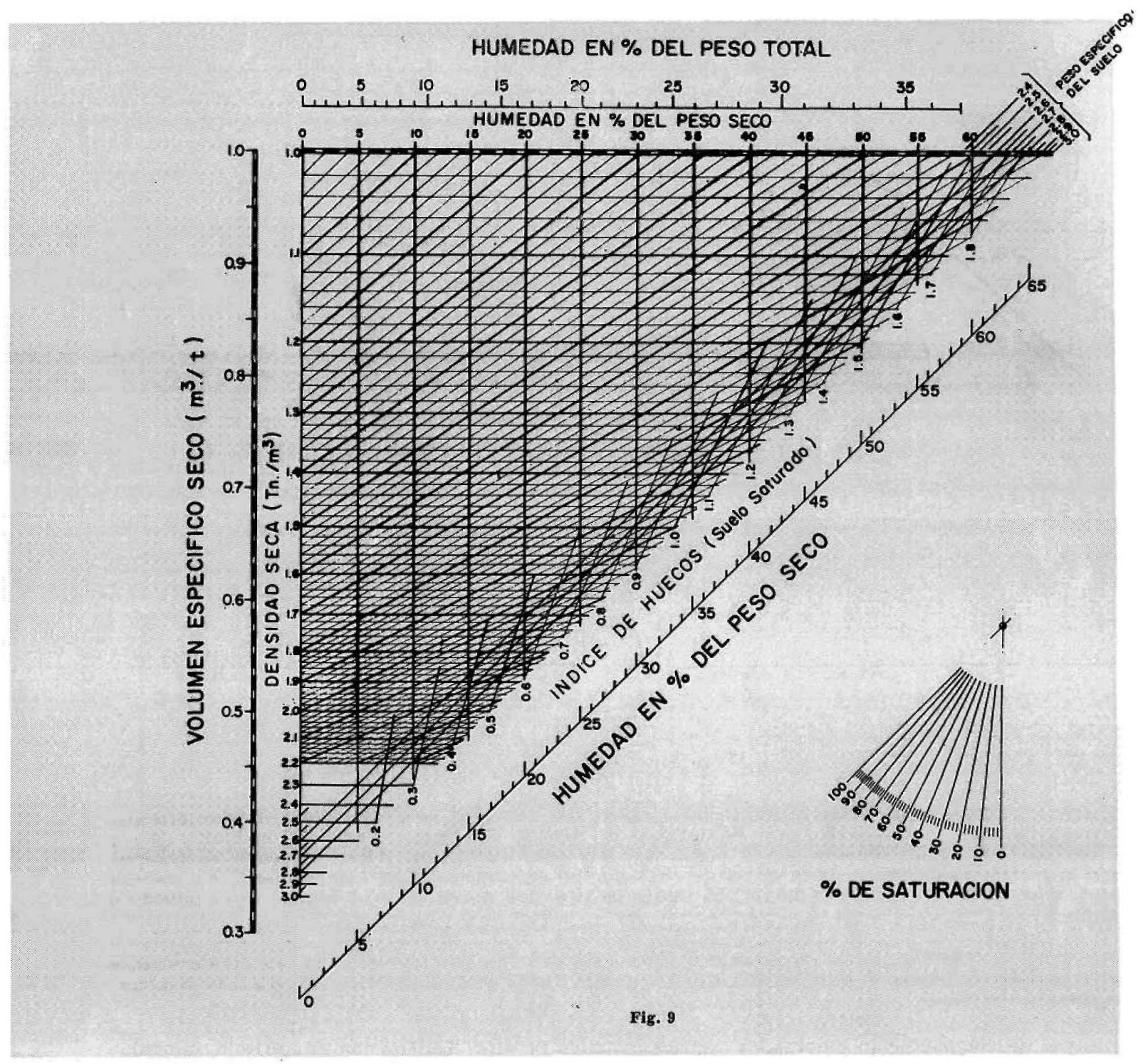

El diagrama de estado del suelo resulta, por lo tanto, una útil herramienta de trabajo tanto para el investigador, al permitirle comparar los resultados de sus experiencias, como para el técnico, que está interesado en controlar la variación del comportamiento de los materiales por medio de ensayos simpliflcados, tales como el de humedad y densidad "in situ" (fig. 9). 


\section{La capacidad de soporte de un terreno}

En el proyecto de firmes, los cuales han de contar, necesariamente, con la colaboración del terreno, se impone un criterio de evaluación de ésta, frente a las deformaciones que causa el tráfico.

En el caso de un firme flexible, el criterio suele basarse en el corrimiento vertical tolerable (total o remanente, como luego se verá) provocado por las cargas que transmiten las ruedas (con interposición o no de las capas suprayacentes a la que se considera: tanto en uno como en otro caso, la huella es semejante, asimilándose, con frecuencia, a un círculo). En el caso de un firme rígido, interesa más la deformabilidad del terreno, o relación entre presiones y corrimientos. Ambos criterioz están relacionados entre sì, y forman parte del mismo fenómeno físico, que se analiza a continuación.

Se denomina "capacidad de soporte" de un terreno a lá máxima presión que éste puede sustentar en su superficie, sin alteraciones graves de su estructura, resultantes en deformaciones intolerables. Si se elige como variable dependiente el corrimiento vertical de la superficie, en el punto correspondiente al centro de la huella de la carga (que, al menos en carreteras, se asimila a un círculo), las variables independientes que tienen una influencia preponderante son la presión aplicada (repartida uniformemente en la huella), el radio de la huella y el número de reiteraciones de la carga.

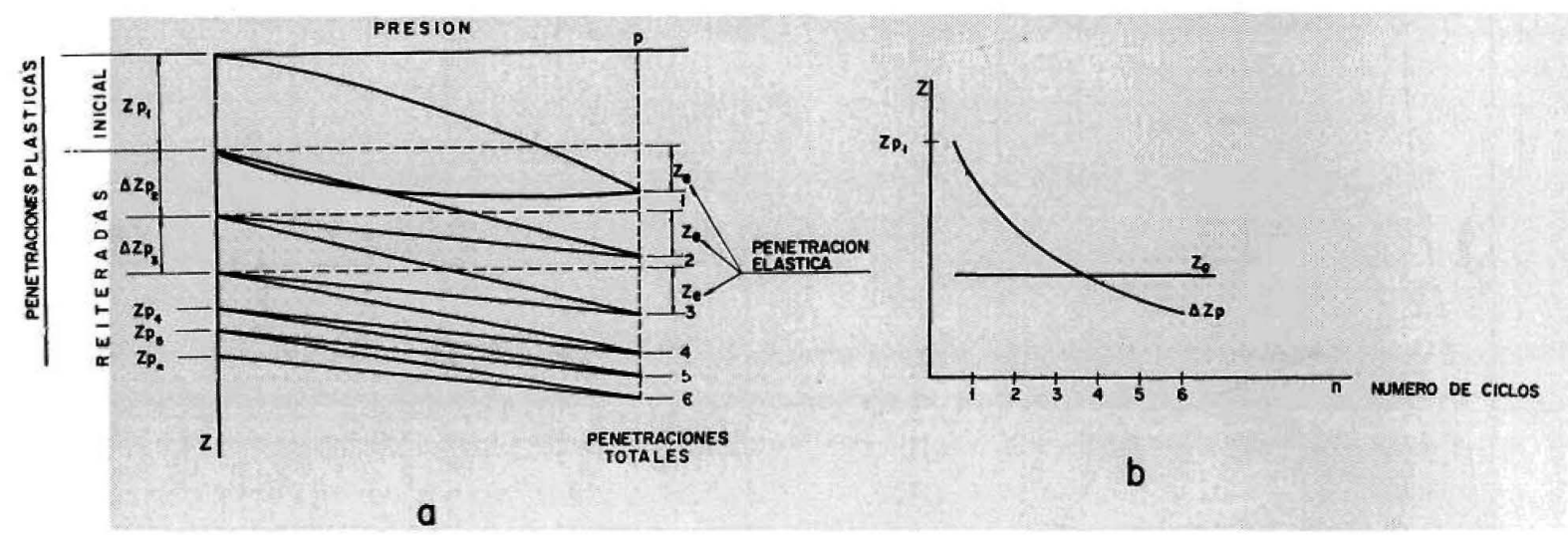

Fig. 10

Si se realiza un ensayo de carga sobre placa circular, aplicando un cierto número, $n$, de veces una presión máxima, $p$, de una forma gradual y durante el tiempo suficiente para que se estabilice la penetración de la placa en el terreno (para prescindir de la influencia de la velocidad de carga, que implicaría una nueva variable), y se traza el diagrama presiones-penetraciones, éste toma un aspecto parecido al de la figura 10a.

Se distinguen inmediatamente dos clases de penetraciones, $z$, o corrimientos de la superficie del terreno:

La penetración elástica, $z_{e}$, que es recuperable en cada ciclo de carga e independiente del número $n$ de éstos.

La penetración plástica o remanente, $z_{\mathrm{p}}$, que decrece al aumentar el número de ciclos (fig. $10 \mathrm{~b}$ ).

En la penetración plástica cabe definir la penetración correspondiente al primer ciclo, $z_{\mathrm{p} 1}$, y los sucesivos incrementos, $\Delta z_{\mathrm{pn}}$, de penetración remanente. Estos últimos, según ha probado la experimentación, decrecen según una ley logarítmica de forma que:

$$
z_{\mathrm{p}}=z_{\mathrm{p} 1}+\gamma \log n
$$

El comportamiento plástico del terreno queda así representado por dos parámetros:

$z_{\mathrm{p} 1}$, que corresponde al asiento inicial y representa el grado de compactación del terreno, antes de la aplicación de las cargas.

, que corresponde a la susceptibilidad del terreno a la fatiga producida por las reiteraciones de la carga.

Dentro de los límites de validez que se definirán más adelante, estos dos parámetros son independientes del radio, a, de aplicación de la carga; mientras que dicho radio tiene una influencia decisiva en la penetración elástica. 
Si se repite el experimento con otro valor de la presión máxima, se determinarán nuevos valores de $\boldsymbol{z}_{t}, \boldsymbol{z}_{, 1}$ y $\gamma ; \mathrm{y}$ así se podrá definir la variación de estos parámetros con $p$. Esta variación es proporcional mientras $p$ no rebase un cierto valor crítico, $p_{e r}$, por encima del cual se produce una redistribución de partículas en el terreno, con fuerte aumento de $z_{\mathrm{p}}$ (flg. 11). Pero por debajo del per es válida la ecuación:

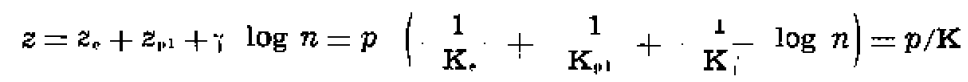

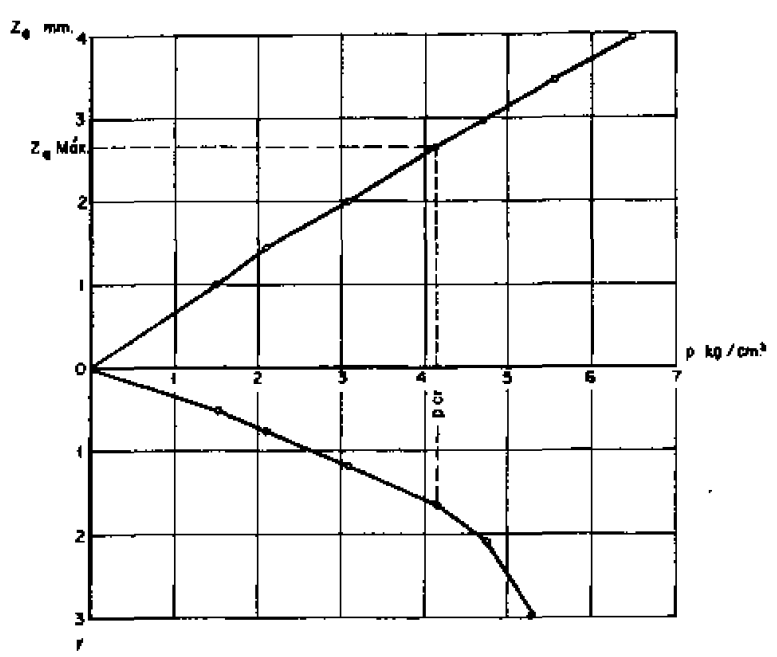

Fis. 11

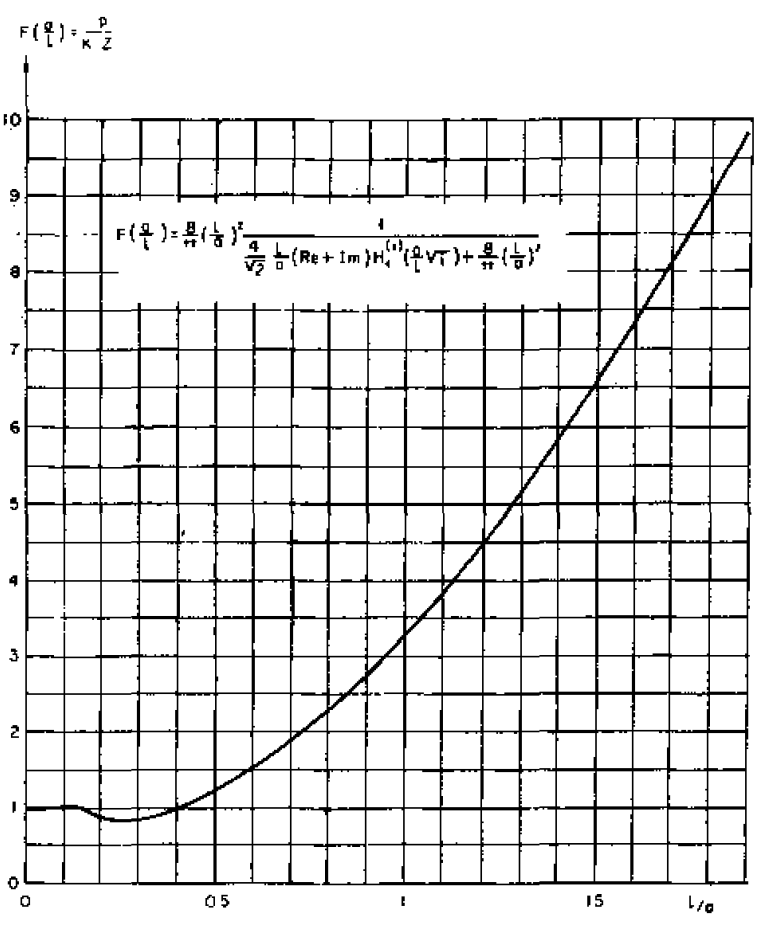

Fig. 12

Los coeficlentes $\mathbf{K}_{s}, K_{\mathrm{p}}$ y $\mathbf{K}_{\mathrm{i}}$ son constantes (dentro del campo de validez antes definido) características del suelo. La única que depende del dímetro de la huella de la carga es $\mathrm{K}$. por lo que conviene definirla en función del mismo, como se verá $a$ continuación. $K_{p}$ depende del estado inicial del terreno, por lo que siempre resulta conveniente aumentarla, mediante una compactación; lo mismo sucede con $K_{Y}$. La $K$ deflnida por la ecuación anterior representa la deformabilidad total del terreno, 0 sea, su "módulo de reacción" o "coeficiente de balasto"; tiene aplicación en el cálculo de tensiones en firmes rígidos.

La variabilidad de $K$. con el dismetro de la carga ha sido objeto de numerosas controversias, hoy día resueltas. Desde antiguo se observó que al aumentar el diámetro de la placa, la relación $1 / \mathbf{K}_{\text {. dis- }}$ minúa asintóticamente a un valor no nulo; a partir de un diámetro de unos $75 \mathrm{~cm}$ (que fue, por lo tanto, elegido como norma para ensayos de carga con placa), el valor de $\mathbf{K}_{\text {a }}$ se estabiliza. Ahora bien: el ensayo con una placa de ese tamaño requiere una reacción considerable, que puede alcanzar las $30 \mathrm{t}$ o más, y no stempre es fácil disponer de ella; por lo que se pensó en extrapolar los resultados de ensayos con placas más pequeñas. En éstas interviene ya la influencia de la resistencia lateral de las zonas no cargadas, contiguas al borde de la carga.

La Teoria de la Elasticidad, y más concretamente Boussinesq, da como valor de $K$, para un semiespacio indefinido de miódulo de elasticidad, $\mathbf{E}$, y de módulo de Poisson, $v$, cargado uniformemente eb un círculo de radio, $a$ :

$$
\mathrm{K}_{0}=\frac{\mathrm{E}}{2 a\left(1-\nu^{2}\right)}
$$

Aparte de la diflcultad de estimar $\mathrm{E} y \vee, \mathrm{K}_{\mathbf{*}}$ no se estabiliza al aumentar $\alpha$; por lo que se buscó simbolizar el comportamiento del terreno en una serie de resortes independientes, de compresibilidad $k$; por lo que, aunque la placa fuera infinitamente grande, seria:

$$
\mathrm{K}_{\mathrm{e}}=\pi
$$


Fsto, a su vez, deja sin cubrir el campo de a pequeños, puesto que no tiene en cuenta la colaboraclón de la periferia. Housel propuso una fórmula lineal con dos parámetros:

$$
\mathbf{K}_{\mathrm{n}}=k+f \frac{\mathbf{P}}{\mathbf{A}}
$$

siendo:

$f=$ Tensión cortante aplicada a lo largo de la circunferencia por la colaboración del terreno circundante.

$\mathbf{P}=$ Perimetro de la huella.

$A=$ Area de la huella.

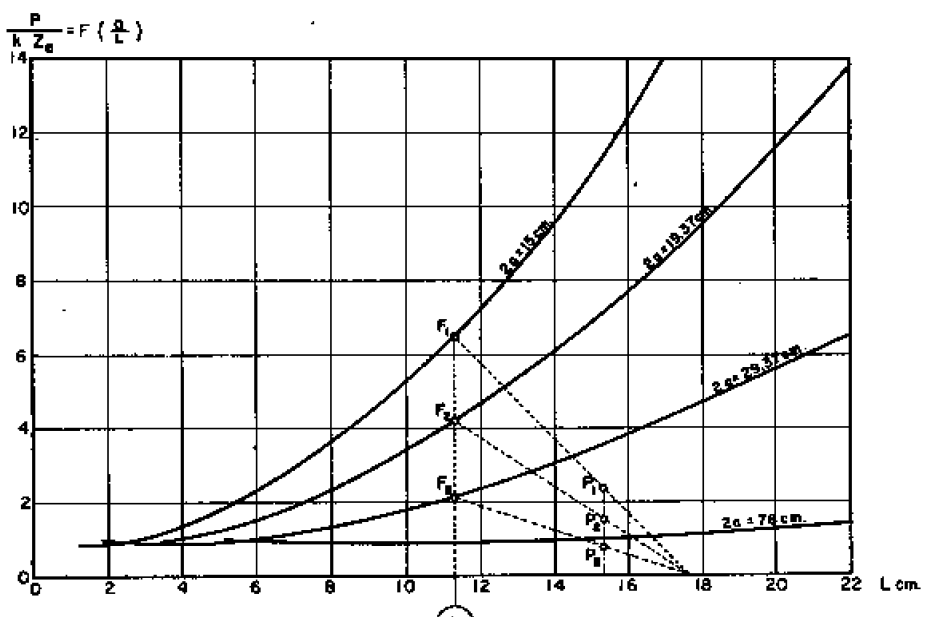

Ffg. 13

Esta fórmula es mejor que la anterior, pero sólo es válida dentro de un intervalo de P/A restringido (aproximadamente, 0,04 a $0,12 \mathrm{~cm}^{-1}$, correspondientes a diámetros de huella de 15 a $50 \mathrm{~cm}$ ), y no representa adecuadamente el fenómeno resistente.

Se requiere, pues, una definición más amplia de la correlación entre $K_{\text {, }}$ y $a$. EI comportamiento del terreno queda más adecuadamente representado si se esquematiza como una losa ficticia, indefinida, caracterizada por un "radio de rigidez relativa", I, flotante sobre un fúido flcticio cuya densidad es $k$, y cargada en un círculo de radio a con una presión $p$. EI corrimiento vertical bajo el centro de la carga viene dado por la expresión:

$$
p=k z \cdot F(a / L)
$$

en la que $F(a / L)$ es una función compleja que ha sido deducida por Maresca en base a los estudios de DeKruyf, Poel y Timman, y se representa en la flgura 12. De ello se deduce que:

$$
\mathrm{K}_{*}=k \mathrm{~F}(a / \mathrm{L})
$$

La determinación experimental de $k$ y $\mathrm{L}$ requiere un mínimo de tres ensayos con placas de diámetros diferentes, y corrimiento elástico, $z_{*}$, constante. Como el terreno, $\mathrm{y}$, por lo tanto, $k \mathrm{y} \mathrm{L}$, es también constante, la presión $p$ necesaria para alcanzar $z_{k}$ es proporcional a $\mathrm{F}(a / \mathrm{L})$, y, por lo tanto, función de $a$. Si se tiene (fig. 13) un diagrama de $F(a / L)$ en función de L para los diversos $a$ utilizados, se pueden situar en él unos puntos de ordenada proporcional a las $p$ obtenidas para diversos a; y por medio de un haz de rectas proyectivas, hallar las $F(a / L)$ que guarden la misma relación proporcional, correspondientes a la misma $\mathrm{L}$. El cálculo de $k$ es entonces inmediato $\mathrm{y}$, consiguientemente, el de $K_{.}$. 
Una vez definido el módulo de reacción del terreno, $K$, en función de las circunstancias especiales del estado del terreno, $\mathbf{K}_{*}$ y $\mathbf{K}_{\mathrm{pl}}, \vec{y}$ del número de reiteraciones, $\mathbf{K}_{\gamma}$, cabe definir la "capacidad de soporte" como aquella presión, $p_{\mathrm{er}}$ rebasada la cual, las deformaciones remanentes aumentan de forma no lineal: $\mathbf{K}_{\mathbf{i}}$ deja de ser constante,

Otras veces, como en el caso de algunos criterios de proyecto de firmes flexibles, se limita aún más el campo, rebajando la $p_{\mathrm{cr}}$ hasta limitar el corrimiento total, $z$, o elástico, $z_{e}$, a un valor máximo fijado por otras consideraciones, tales como rodadura, etc.

Tanto en un caso como en otro, los parámetros $\mathbf{K}_{t}, \mathbf{K}_{\mathbf{p} 1}$ y $\mathbf{K}_{\mathbf{i}}$ son constantes que dependen del estado inicial del terreno.

\section{El rendimiento de un terreno como soporte}

Definida como anteriormente la capacldad de soporte de un terreno, y conseguido su valor máximo (compatible con la economia) por medio de una compactación adecuada, puede que dicha capacidad resulte todavía insuficiente para los fines a que se destine el terreno: por ejemplo, a soportar las cargas del tráfico (prescindiendo de consideraciones de durabilidad que obligarían a disponer una capa de rodadura). Resulta obligado entonces disponer una capa de otro terreno cuya capacidad resistente sea mayor 0 , incluso, de varias capas.

Es evidente que el espesor de dicha capa adicional tiene influencia sobre la capacidad de soporte del conjunto. En efecto, si la capa adicional es miy delgada, la capacidad resistente será prácticamente igual a la del terreno subyacente; mientras que si es muy espesa, la influencia de este último se verá $\tan$ atenuada, adquiriendo el conjunto una capacidad correspondiente a un macizo inflnito de la capa de aportación. Entre uno y otro extremo se sitúa la solución económica, que es función del "rendimiento) que se define a continuación.

Se ha comprobado experimentalmente que para una capacidad resistente, $p_{u}$, en el terreno subyacente (que a su vez puede estar formado por una o varias capas), al disponer encima de él una capa de espesor, $e_{s}$ de un terreno de capacidad resistente (en capa infinita), $p_{l}$, la capacidad aumenta según Ia ley logaritmica:

$$
p=p_{0}+\lambda \log e
$$

en la cual $\lambda$ es el "rendimiento", que tiene las mismas dimensiones que $p$. Este rendimiento no es constante, sino que cisminuye al aumentar $p_{0}$ anulánáose para $p_{0}=p_{1}$. Esta disminuclón se verlfica de forma casi lineal (fig. 14), a partir de un cierto valor de $p_{0}$ al que corresponde un máximo de $\lambda$. De esto se deduce que no es conveniente rebasar en el terreno o capa subyacente a una dada, la capacidad de soporte que en el diagrama $p_{0}-\lambda$ corresponde a un rendímiento máximo de la capa en cuestión; pues dicho rendimiento, o sea, la capacidad de aumento de soporte con el espesor, baja rápidamente si se aumenta $p_{0}$.

Así, se puede concebir el dimensionamiento económico del soporte de un firme (flexible o rigido) de la siguiente manera:

Datos de partida:

Un terreno natural.

Unos materiales de aportación, deflnidos por sus curvas de rendimiento. Entre ellos pueden contarse el propio terreno natural, debidamente compactado o estabilizado.

Una capacidad de soporte en la última capa, o un módulo de reacción del conjunto, exigidos por el firme. En el primer caso, se va a disponer un firme tlexible; en el segundo, uno rígida.

Se pueden elegir varias combinaciones de materiales de soporte, y seguir con ellas el criterio expuesto en la figura 15. 
Este criterio consiste en tantear los costos de diversas combinaciones de materiales de aportación, pero con la condición de que la capacidad de cada capa sea la que corresponde a un rendimiento óptimo de la superior, y su espesor aquel que determina la condición anterior, y la capacidad de la capa inferior. El coste de cada capa viene influido por su espesor, y el coste total comprende el de compactación del terreno natural hasta la capacidad que proporciona un rendímiento óptimo de la capa inmediatamente superior.

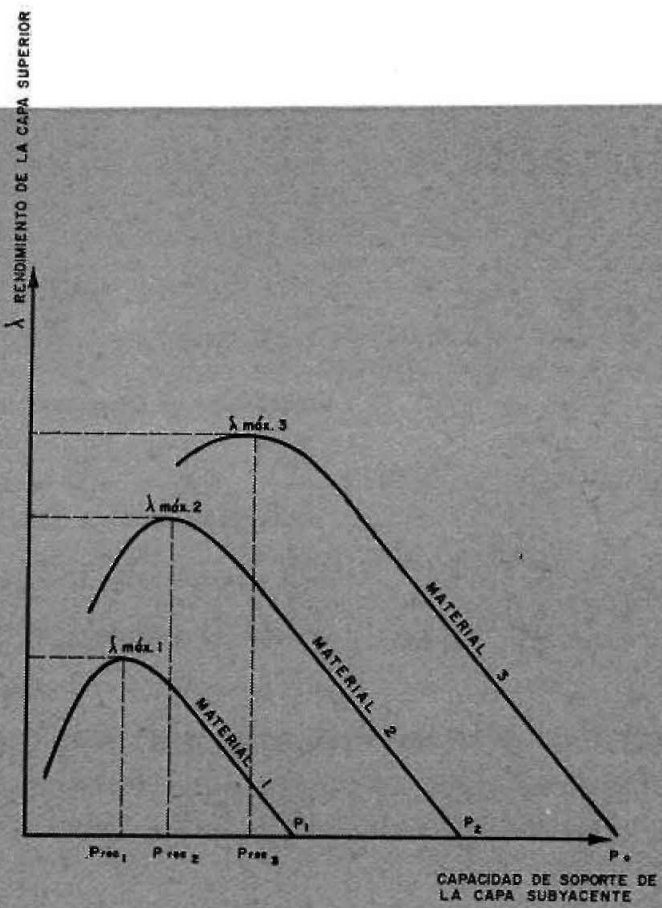

Fig, 14
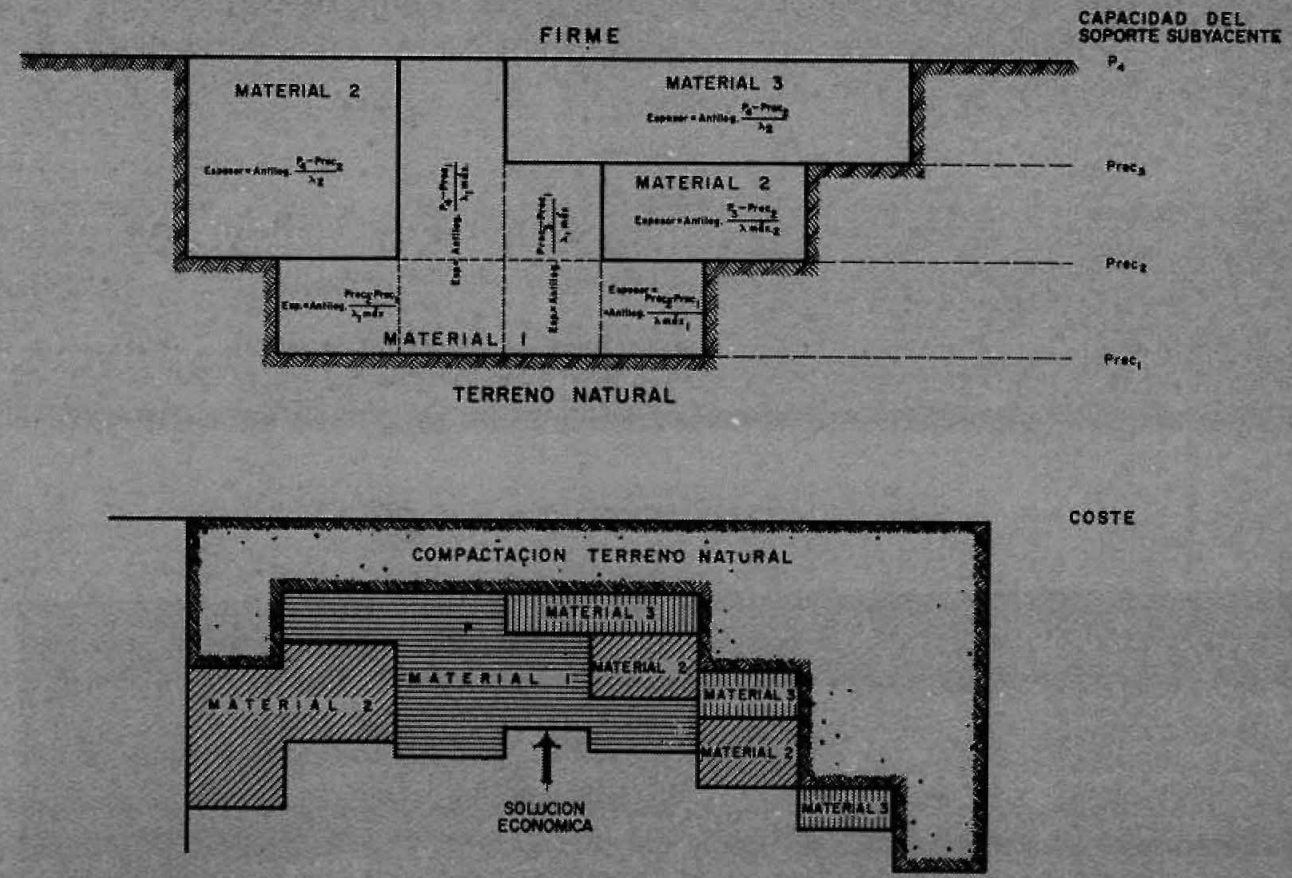

coste

Fig. 15 